\title{
XB130 is overexpressed in prostate cancer and involved in cell growth and invasion
}

\author{
Bin Chen ${ }^{1,2, *}$, Mengying Liao ${ }^{3, *}$, Qiang Wei ${ }^{4, *}$, Feiye Liu ${ }^{5}$, Qinsong Zeng ${ }^{6}$, Wei Wang ${ }^{6}$, \\ Jun Liu', Jianing Hou ${ }^{7}$, Xinpei $\mathbf{Y u}^{8,9}$, Jian Liu ${ }^{9}$ \\ ${ }^{1}$ Department of Science and Training, General Hospital of Guangzhou Military Command of People's Liberation Army, \\ Guangzhou, Guangdong, China \\ ${ }^{2}$ Guangzhou Huabo Biopharmaceutical Research Institute, Guangzhou, Guangdong, China \\ ${ }^{3}$ Department Of Pathology, Peking University Shenzhen Hospital, Shenzhen, China \\ ${ }^{4}$ Department of Urology, Nanfang Hospital, Southern Medical University, Guangzhou, Guangdong, China \\ ${ }^{5}$ Cancer Center, Traditional Chinese Medicine-Integrated Hospital of Southern Medical University, Guangzhou, Guangdong, \\ China \\ ${ }^{6}$ Department of Urology, General Hospital of Guangzhou Military Command of People's Liberation Army, Guangzhou, \\ Guangdong, China \\ ${ }^{7}$ Sun Yat-Sen University, Guangzhou, China \\ ${ }^{8}$ Guangdong Provincial Key Laboratory of Geriatric Infection and Organ Function Support and Guangzhou Key Laboratory of \\ Geriatric Infection and Organ Function Support, Guangzhou, Guangdong, China \\ ${ }^{9}$ Center for Geriatrics, General Hospital of Guangzhou Military Command of People's Liberation Army, Guangzhou, Guangdong, \\ China \\ *These authors contributed equally to this work
}

Correspondence to: Xinpei Yu, email: yxinpei@126.com Jian Liu, email: liujgzzyy@126.com

Keywords: XB130, adaptor protein, proliferation, invasion, Akt

Received: January 27, $2016 \quad$ Accepted: June 29, 2016

Published: August 05, 2016

\section{ABSTRACT}

XB130 is a cytosolic adaptor protein involved in various physiological processes and oncogenesis of certain malignancies, but its role in the development of prostate cancer remains unclear. In current study, we examined XB130 expression in prostate cancer tissues and found that XB130 expression was remarkably increased in prostate cancer tissues and significantly correlated with increased prostate specific antigen (PSA), free PSA (f-PSA), prostatic acid phosphatase (PAP) and T classification. Patients with highly expressed XB130 had significantly decreased survival, which suggested XB130 as a possible prognostic indicator for prostate cancer. In vitro experiments showed that reduced XB130 expression restrained tumor growth both in vitro and in vivo. Furthermore, XB130 knockdown hindered transition of G1 to $S$ phase in prostate cancer cell line DU145 and LNCap, which might contribute to the inhibition of cellular proliferation. Results from transwell assay demonstrated that downregulation of XB130 may attenuate invasion and metastasis of prostate cancer. Semiquantitative analysis of Western blot suggested that decreased XB130 expression was accompanied by diminished Akt signaling and EMT process. Thus, above observations suggest that XB130 may be a novel molecular marker and potent therapeutic target for prostate cancer.

\section{INTRODUCTION}

Prostate cancer is the most common and second lethal cancer in western male population $[1,2]$. PSA is the major significant marker for the diagnosis of prostate cancer with a low specificity of $18.67 \%-30.08 \%$ as single use in for biopsy [3]. To clarify the molecular mechanism involved in prostate cancer is of great importance for finding a potent and more effective diagnostic marker and improving the treatment of prostate cancer. 
Adaptor protein has a unique modular structure without enzymatic activity, which can be regulated by multiple signaling. It consists of actin filament associated protein (AFAP), Src interacting/Signal integrating protein (Sin) and Crk-associated substrate (CAS). Multiple studies have proved this functions in mitosis, differentiation, inflammation, cell survival, movement, and adhesion through binding SH3 and SH2 domains and activating c-Src [4-6]. AFAP, as a small adaptor protein, is involved in cell signal transduction, assembling of cell cytoskeleton and other cell functions, which can directly induce activation of c-Src by mechanical stretch. XB130, also named AFAP1L-2, is a $130 \mathrm{kDa}$ adaptor protein with 818 amino acids located on chromosome 10q25.3 [7]. As the substrate and regulator of signal transduction mediated by tyrosine kinases, XB130 phosphorylates tyrosine through $\mathrm{SH} 2$ and $\mathrm{SH} 3$ domains at the N-terminal of Src [8]. The role of XB130 varies in cell proliferation, survival, movement and invasion in different malignancies [9-11]. In thyroid carcinoma, aberrant XB130 expression induces cell death and enhances apoptosis [12]. Interestingly, high XB130 protein level improves survival and sensitivity to 5-Fluorouracil in gastric cancer patients [13] while correlates with higher pathological grade and poorer prognosis of pancreatic ductal carcinoma [14]. Thus the association between XB130 and prostate cancer are worthy to be explored. Our previous findings demonstrated that low to moderate XB130 expression were seen in normal, hyperplastic and peritumoral prostate tissues, while elevated XB130 expression was displayed in prostate cancer tissues (Data not shown), but whether XB130 can affect cellular growth and invasion in prostate cancer is unclear. Thus in present study, we further investigated the linkage between $\mathrm{XB} 130$ and prognosis of prostate cancer and effects of $\mathrm{XB} 130$ on proliferation, invasion and metastasis of prostate cancer cells.

\section{RESULTS}

\section{$\mathrm{XB130}$ is a novel prognostic indicator for prostate cancer}

We first examined XB130 expression in 210 cases of prostate cancer with pathological diagnosis. Consistent with our previous findings (data not shown), XB130 expression was remarkably increased in prostate cancer tissues as compared with hyperplastic and normal tissues (Figure 1A). As Table 1 indicated, XB130 was positively expressed in $85.6 \%$ specimens and significantly correlated with increased prostate specific antigen (PSA) $(p=0.006)$, free PSA (f-PSA) $(p=0.036)$, prostatic acid phosphatase (PAP) $(p=0.02)$ and T classification $(p=0.025)$. KaplanMeier analysis (Figure 1B) revealed that patients with highly expressed XB130 have significantly decreased survival rate than those with lower XB130 expression $(p=0.0121)$, which indicated that XB130 expression was inversely correlated with survival in prostate cancer.

\section{XB130 knockdown suppresses growth of prostate cancer in vitro}

XB130 mRNA (Figure 2A) and protein (Figure 2B) levels were detected in prostate cancer cell line 22RV1, LNCap, DU145, PC3, which showed that XB130 expressed in four prostate cancer cell lines at different levels. DU145 and LNCap expressed more XB130 in four prostate cancer cell lines than 22RV1 and PC3, thus were chosen for further knockdown studies.

To explore the effect of XB130 on proliferation of prostate cancer, we established two cell lines with downregulated XB130 expression and named as DU145/ XB130- and LNCap/XB130-, while control cell lines were DU145/mock and LNCap/mock (Figure 2C). CCK8 assay (Figure 2D) showed that proliferation of DU145/ XB130- was significantly slower than DU145/mock cells $(\mathrm{F}=34.988, p=0.02)$. Similar disparity was seen in LNCap/XB130- cells (Figure 2D, $\mathrm{F}=43.644, P=0.000$ ). Colony formation assay (Figure $3 \mathrm{~A}$ and $3 \mathrm{~B}$ ) indicated that, knockdown of XB130 in DU145 $(\mathrm{F}=95.663, p=0.000)$ and LNCap $(\mathrm{F}=92.789, p=0.000)$ could suppress the formation of colony of both groups comparing to DU145/ mock and LNCap/mock. Downregulation of XB130 resulted in repression of colony formation by nearly $84 \%$ and $89 \%$ in DU145 and LNCap, respectively. Thus, decreased XB130 led to inhibitive proliferation of prostate cancer cell lines, which supported our previous conclusion that strong XB130 expression enhanced the growth of prostate cancer. In flow cytometry analysis (Figure 3C and 3D), DU145/XB130- (23.05\% vs $26.92 \%$, $p=0.04)$ and LNCap/XB130- $(18.86 \%$ vs $24.12 \%$, $p=0.03$ ) displayed strikingly shorter $\mathrm{S}$ phase than control cells. Although not statistically significant, G1 phase prolongation were shown in DU145/XB130- $(66.16 \%$ vs $56.43 \%, p=0.2)$ and LNCap/XB130- cells $(70.45 \%$ vs $65.43 \%, p=0.3$ ), which suggested that decreasing XB130 might subdue the transition of G1 to S phase in prostate cancer, which might be a cause for the restrain of cellular proliferation.

\section{XB130 knockdown inhibits tumor growth in vivo}

We also investigated the effect of XB130 on the tumor growth using xenograft model in nude mice. Six days after subcutaneous injection of $10^{7}$ cells on the back of nude mice, we started to assess the tumor growth by measuring the tumor size when apparent tumors were seen on the back of mice. The tumor formation in the XB130 knockdown groups was significantly slower than in control groups. The tumors of knockdown groups were strikingly smaller when compared with control groups at day 18 and the difference became significant at day 21 after injection. As indicated in Figure 4A and 4B, remarkable suppression of tumor size and volume was seen in xenografts from DU145/XB130$(p=0.03)$ and LNCap/XB130- $(p=0.02)$ when compared 
Table 1: Relation between XB130 expression and clinicopathologic variables inprostate cancer patients

\begin{tabular}{|c|c|c|c|c|c|}
\hline variables & low expression & high expression & total & $t / \chi^{2}$ & $P$ \\
\hline gleason & $6.24 \pm 1.84$ & $7.04 \pm 1.51$ & & -3.295 & 0.001 \\
\hline PSA & $43.08 \pm 68.68$ & $79.39 \pm 143.15$ & & & 0.006 \\
\hline f-PSA & $8.71 \pm 13.70$ & $15.20 \pm 22.80$ & & & 0.036 \\
\hline \multicolumn{6}{|l|}{$\mathrm{T}$ classification } \\
\hline 1 & $11(17.2 \%)$ & $8(5.5 \%)$ & $19(9.1 \%)$ & 9.356 & 0.025 \\
\hline 2 & $37(57.8 \%)$ & $102(70.3 \%)$ & $139(66.5 \%)$ & & . \\
\hline 3 & $8(12.5 \%)$ & $11(7.6 \%)$ & $19(9.1 \%)$ & & . \\
\hline 4 & $8(12.5 \%)$ & $24(16.6 \%)$ & $32(15.3 \%)$ & & . \\
\hline \multicolumn{6}{|l|}{ PAP } \\
\hline 1 & $6(9.7 \%)$ & $4(2.7 \%)$ & $10(4.8 \%)$ & 7.866 & 0.02 \\
\hline 2 & $16(25.8 \%)$ & $24(16.4 \%)$ & $40(19.2 \%)$ & & . \\
\hline 3 & $40(64.5 \%)$ & $118(80.8 \%)$ & $158(76.0 \%)$ & & . \\
\hline
\end{tabular}

A

Nomal

Hyperplasia

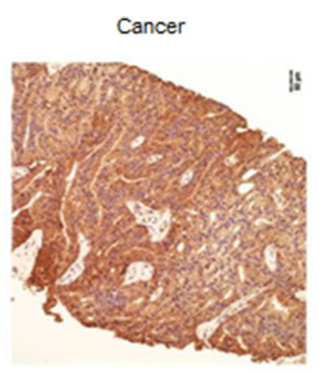

$200 x$
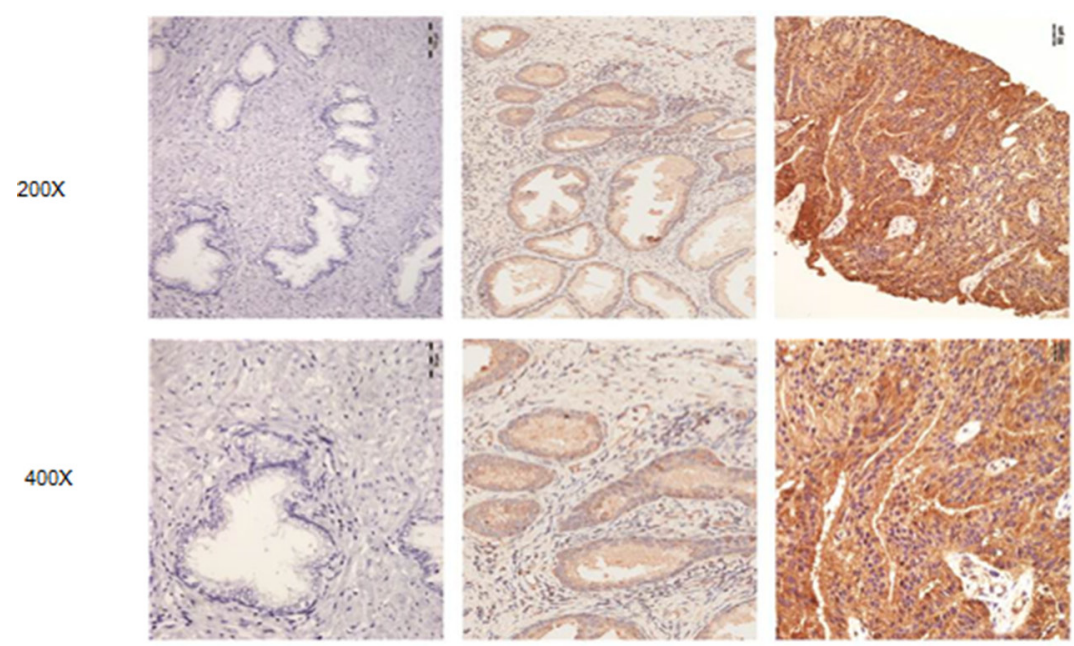

B

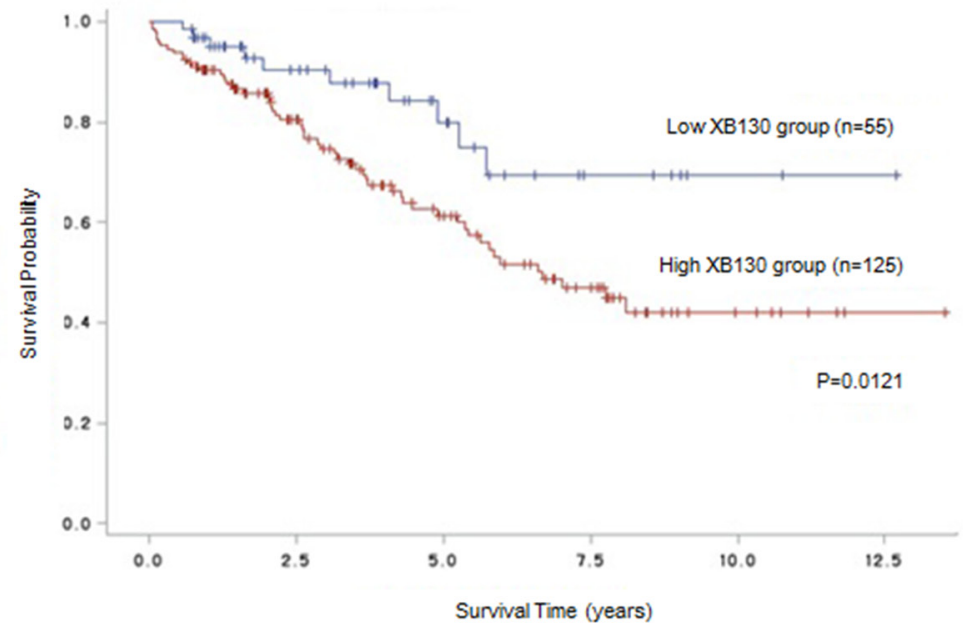

Figure 1: XB130 expression was correlated with prognosis of prostate cancer. (A) Representative IHC-stained section of XB130 in different prostate tissues. XB130 expression was remarkably increased in prostate cancer tissues as compared with hyperplastic and normal tissues. (B) Kaplan-Meier analysis of the relation between XB130 and prognosis of prostate cancer. 
with control groups. In DU145/XB130- and LNCap/ XB130- groups, the average tumor volumes reached 0.07 and $0.06 \mathrm{~cm}^{3}$ on day 24 after tumor cell inoculation, whereas control groups had average volumes of 0.37 and $0.43 \mathrm{~cm}^{3}$ (Figure 4C and 4D). These findings suggested that decreased XB130 restrained tumor growth in vivo.

\section{XB130 knockdown attenuates invasiveness of prostate cancer}

To determine whether XB130 affects invasion of prostate cancer, we performed transwell assay with DU145 and LNCap cells. As shown in Figure 5A-5D, knockdown of $\mathrm{XB} 130(\mathrm{~F}=83.922, P=0.01)$ significantly inhibited invasion
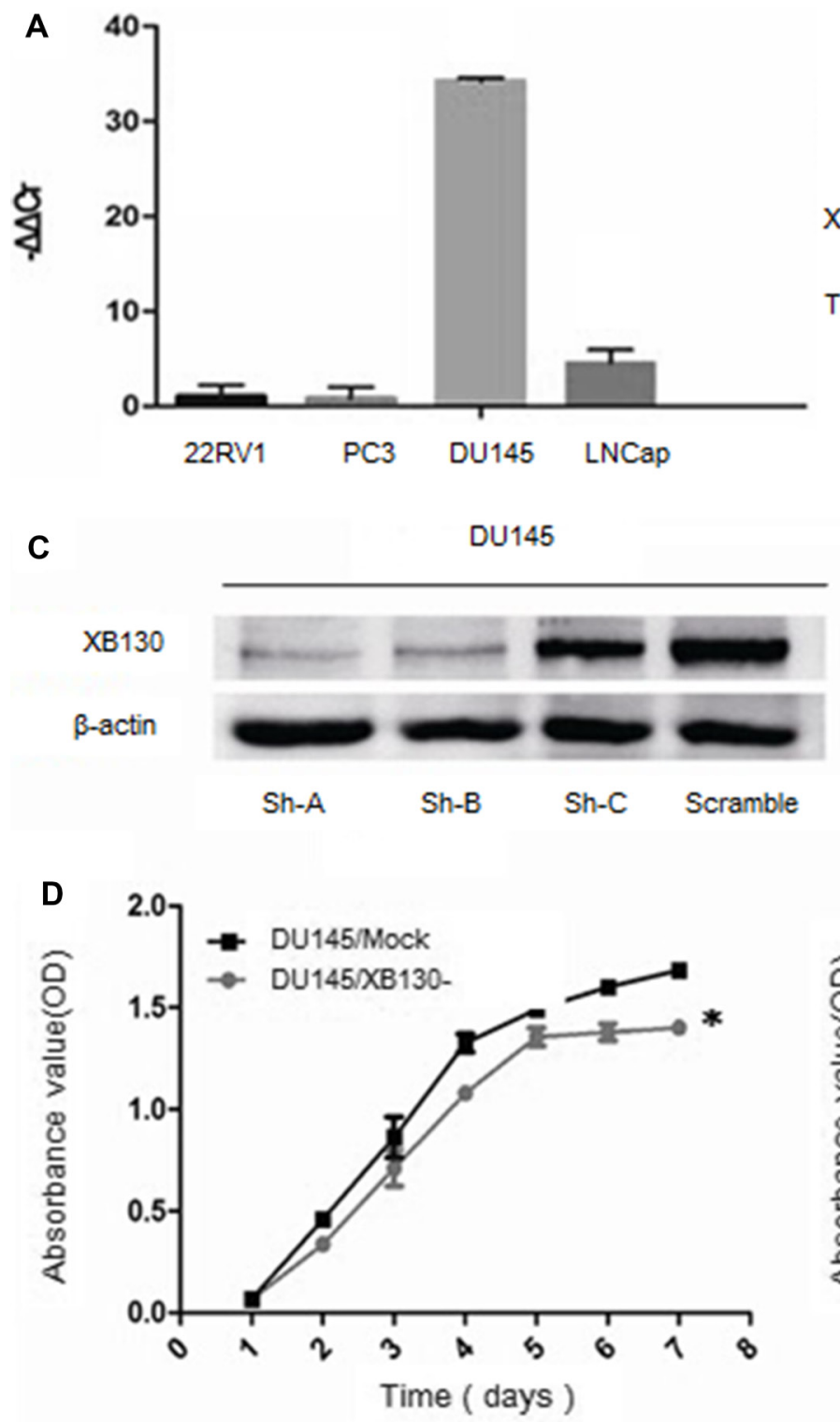

in DU145 cells when compared with control cells. Similar difference was also shown in LNCap cells $(\mathrm{F}=92.164$, $P=0.002)$. These data implied that endogenous XB130 also boosted invasion and metastasis of prostate cancer.

\section{XB130 stimulates Akt and EMT signaling}

In order to pursue the mechanisms of XB130 on the tumor progression, we detected potential downstream targets of XB130. Semiquantitative analysis of western blot (Figure 6A and 6B) showed that p-PDK1, p-C-Raf and Thr308 protein levels in DU145/XB130- and LNCap/ XB130 - significantly decreased, while p-PTEN was upregulated as compared to control cells. Interestingly,

B
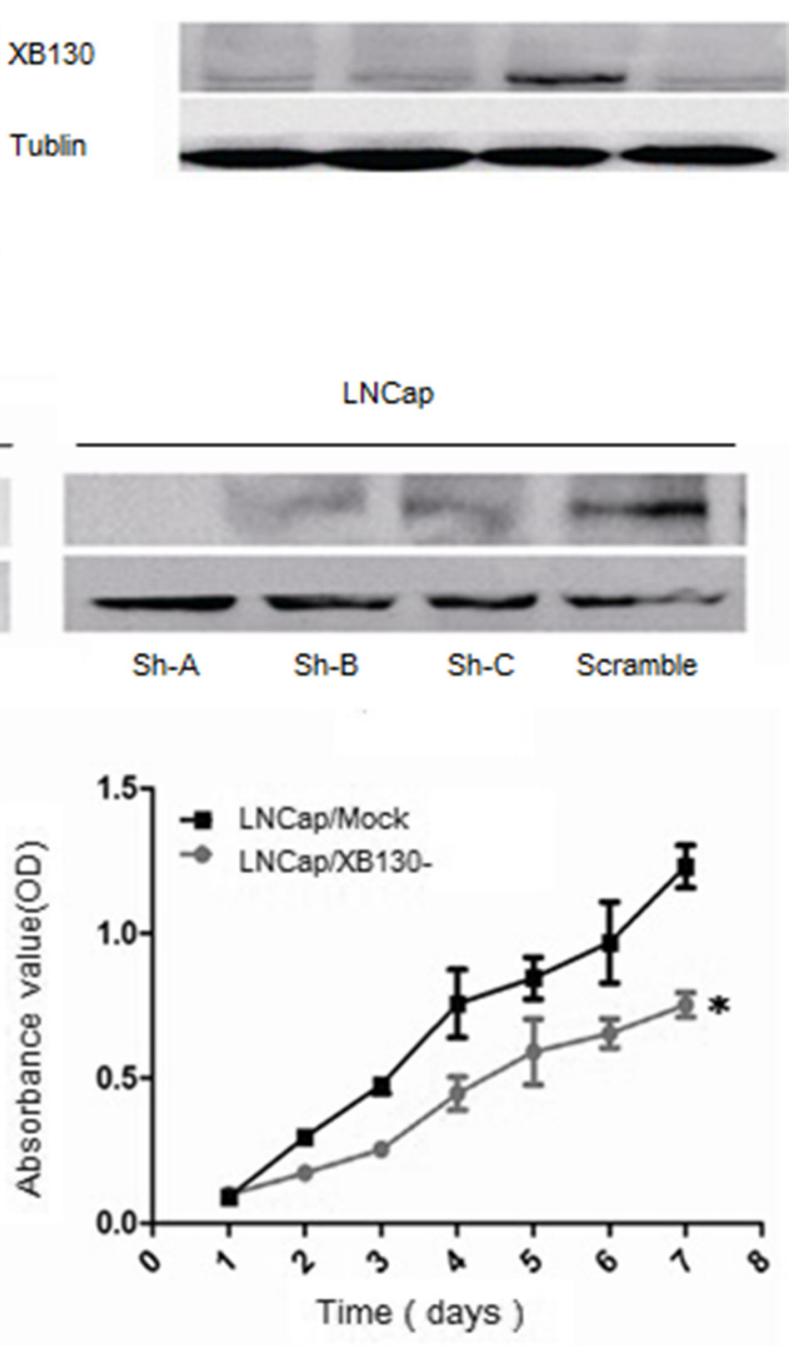

Figure 2: Knockdown of XB130 inhibited the proliferation of prostate cancer cell lines. XB130 mRNA (A) and protein (B) expression in different prostate cancer cell lines. (C) Downregulation of XB130 with shRNA in DU145 and LNCap cells. (D) CCK8 assay indicated that XB130 knockdown inhibited cellular growth in DU145 and LNCap. Data are represented as mean $+/-$ SEM. $* P<0.05$ as compared to control groups. 
AKT (pan) expression didn't exhibit difference inXB130 knockdown cells. Two well-known markers of epithelialmesenchymal transition (EMT) were also affected by XB130 abatement, with mesenchymal marker vimentin expression increased and epithelial marker E-cadherin decreased (Figure 6C and 6D).

\section{DISCUSSION}

At present, only a few studies investigated the role of XB130 in cancer. In an immunohistochemistry analysis of 52 samples, $71.2 \%$ of the patients expressed high levels of XB130 in esophageal squamous cell carcinoma tissues, indicating that it may be a possible biomarker for diagnosis of esophagus cancer. It has also been proved to be an independent poor prognostic factor for survival of patients after operation [15]. Upregulation of XB130 and its significant connection with TNM stage and tumor differentiation were found in pancreatic cancer and breast cancer $[16,17]$. But in gastric cancer, the expression profile seemed to be opposite. Low expression of XB130 was accompanied by shorter survival, disease-free period and diminished response to 5-fluorouracil therapy [13]. According to our results, XB130 level elevated in prostate cancer tissues when compared with adjacent tissues and $85.6 \%$ specimen shows positive staining, which suggests that XB130 could be a candidate marker assisting diagnosis of prostate cancer. Moreover, enhanced XB130 was correlated with a lower survival rate, which indicates it may serve as a poor prognostic factor in prostate cancer. The functions of XB130 in other cancers are worthy of more inspection.

Then we explored the possible effects and underlying mechanism of XB130 on prostate cancer cells. Several reports had demonstrated XB130 is involved in modulating proliferation and survival through regulation
A
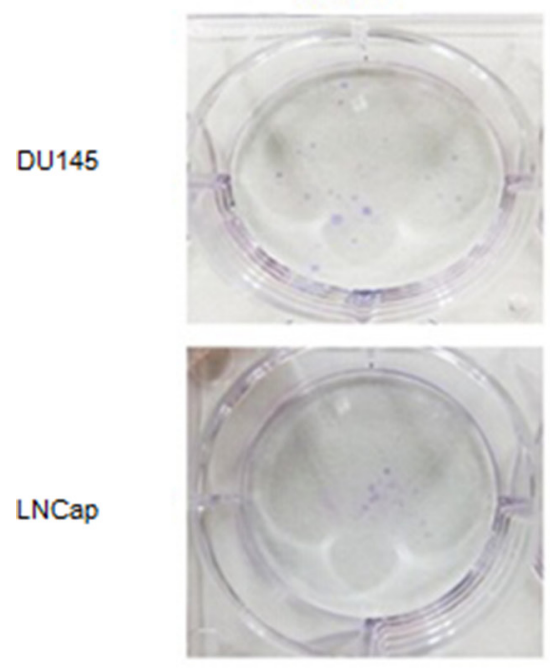

Mock
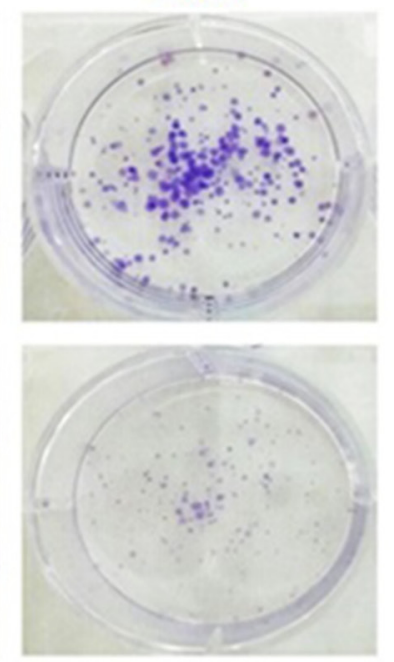

B
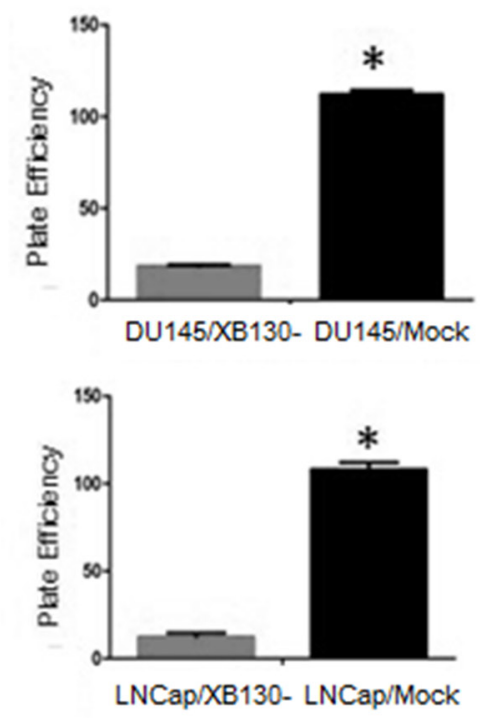

C

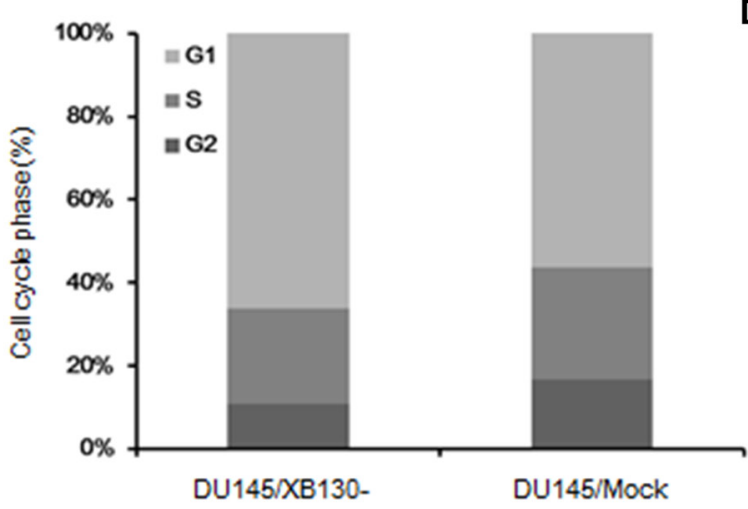

D

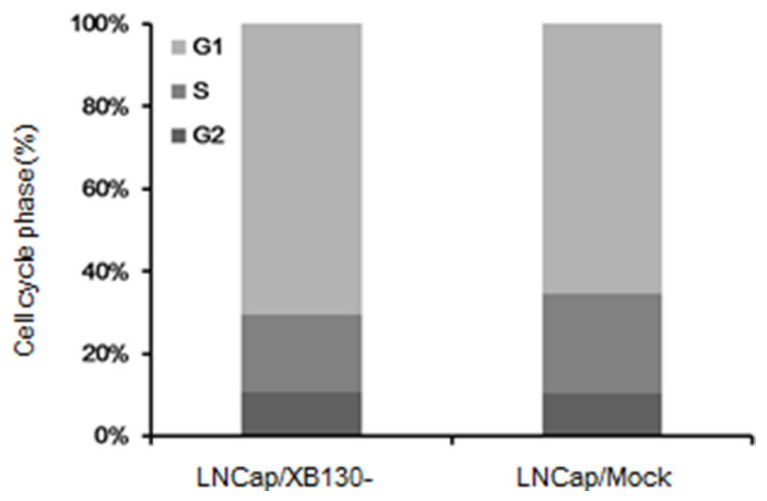

Figure 3: XB130 may restrain cellular proliferation through modulate transition of $\mathrm{G} 1$ to $\mathrm{S}$ phase in prostate cancer. (A) XB130 knockdown decreased the colony formation of DU145 and LNCap. (B) Quantitative analysis of the colony form assay. Data are represented as mean $+/-$ SEM. $* P<0.05$ as compared to the control groups. (C) Flow cytometry analysis of cell cycle changes in DU145/ XB130- and LNCap/XB130-. DU145/XB130- and LNCap/XB130- had significant shortened S phases than corresponding control groups. XB130 knockdown led to G1 phase arrest in both DU145 and LNCap. 
of essential cellular signaling [9-11]. Through RET/PTC pathway in thyroid cancer cells, XB130 diminution results in spontaneous apoptosis and enhancement of cell death with stimulant [11]. Downregulation of XB130 in thyroid cancer leads to remarkable alteration in gene expression profiling, including 57 genes involved in cell growth or survival and modulation of transcription. Pathway analysis suggests that the main molecular and cellular function of XB130 is modulation of proliferation, viability and cell cycle. Most common aliments associated with dysfunction of XB130 are all related to cancer [10]. Besides, inhibition of endogenous XB130 expression impairs the activity of c-Src, IL-8 signalizing and Akt phosphorylation in lung cancer cells [7]. Above findings indicates a vital role of
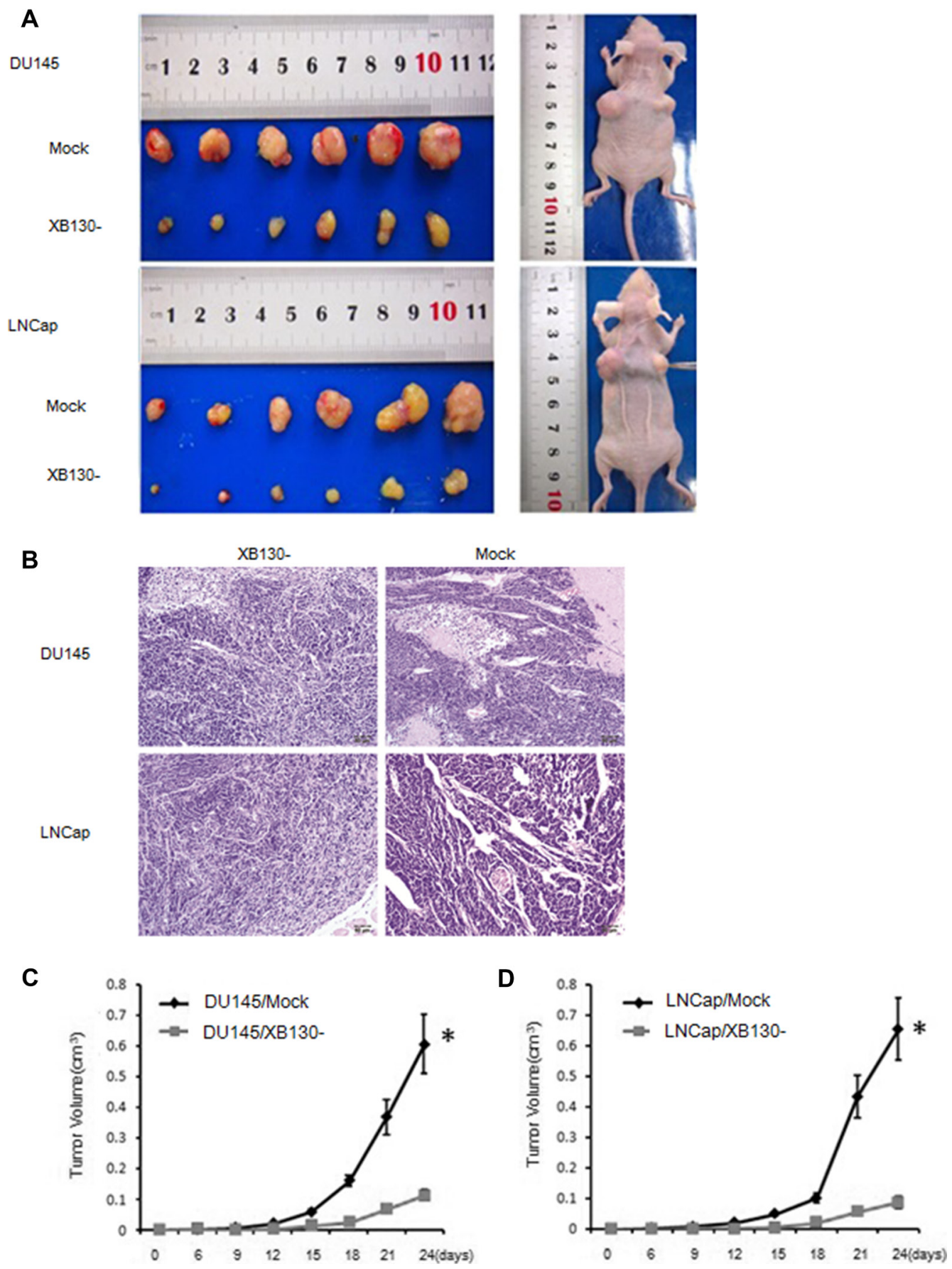

Figure 4: XB130 downregulation inhibited prostate cancer growth in vivo. (A) DU145/XB130-and LNCap/XB130- tumors showed significant decreases in tumor volume comparing with control tumors on day 24 after injection. (B) Histology of DU145/XB130and LNCap/XB130- tumors. Magnification, $\times 200$ (C) Tumor growth curve of after injection of nude mice with DU145/XB130- or control cells. (D) Tumor growth curve of after injection of nude mice with LNCap/XB130- or control cells. Data are represented as mean +/- SEM. $* P<0.05$ as compared to control groups. 
XB130 in the growth, and survival of certain malignancies. Thus, we examined if XB130 also affect development of prostate cancer which could provide evidence for it being a candidate molecular target of novel therapy. CCK8 assay performed at different time points indicated that decreased XB130 remarkably attenuated viability of prostate cancer cells. Colony formation assay also supports this assumption by the result that XB130 knockdown caused a strikingly reduction in the number of colonies formed in DU145 and LNCap. Moreover, XB130 downregulation were accompanied by a diminished effect on S-phase cells and increased proportion of G1-phase cells, which is in line with previous findings in other solid tumors like lung cancer, thyroid cancer and esophagus cancer [7, 9, 10, 15]. Therefore, XB130 is an essential factor for proliferation

A

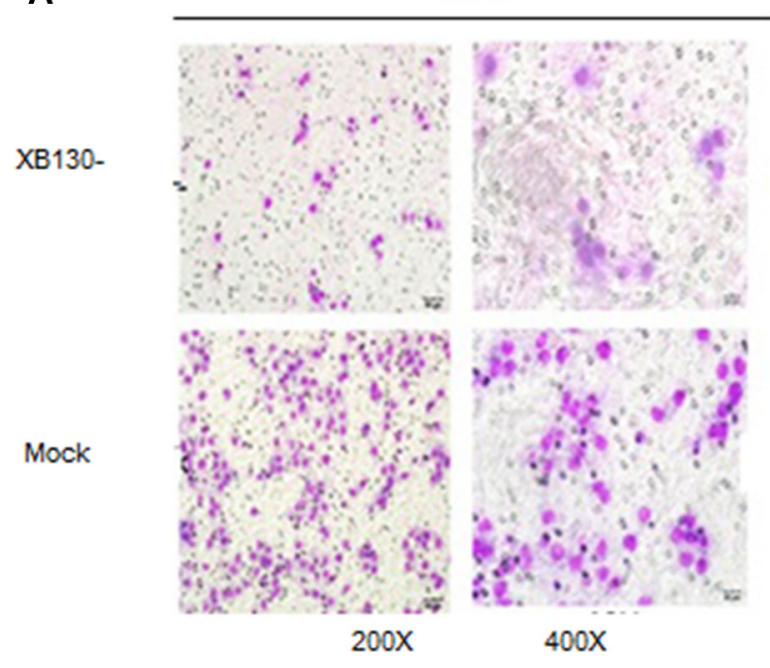

C

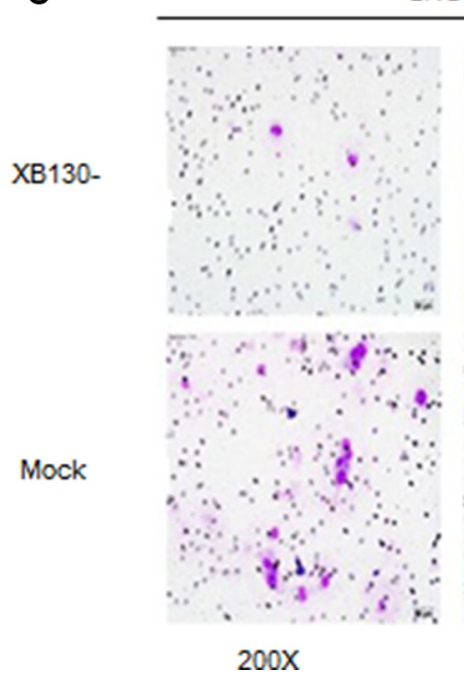

DU145

LNCap

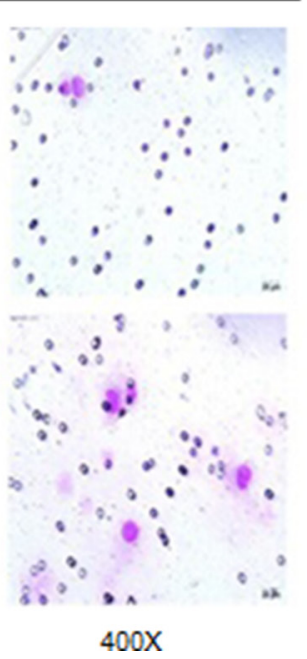

of prostate cancer in vitro and G1 phase changes may contribute to this process. Further study on xenografts confirmed that XB130 knockdown inhibits growth of prostate cancer cells. Thus, XB130 overexpression may promote development of prostate cancer.

It has been reported that overexpression of XB130 boosts nicotine-derived nitrosamine ketone(NNK)induced bronchial epithelial cell migration through enhancement of tyrosine phosphorylation and matrix metalloproteinase-14 translocation [18]. High-affinity lamellipodial F-actin reticular enables XB130 to take part in migration and invasion of thyroid cancer cells in a stimulus-induced fashion [7]. In this study, interference of XB130 significantly weakened the invasiveness of DU145 and LNCap, which suggested

\section{B}

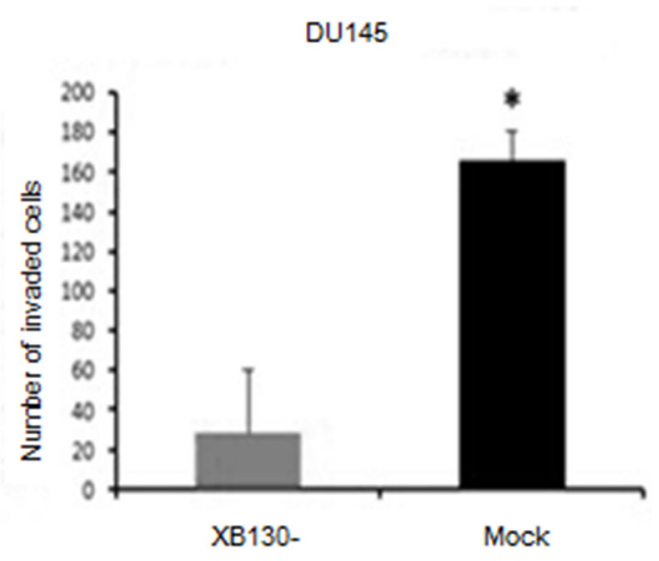

D

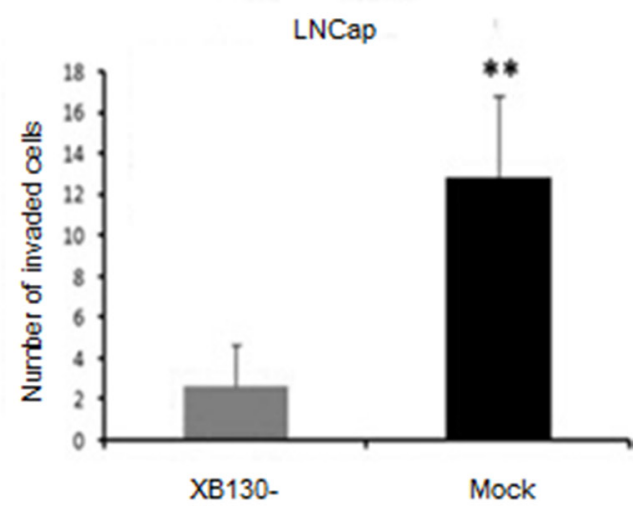

Figure 5: Reduced XB130 expression attenuated invasiveness of prostate cancer. (A) XB130 downregulation inhibited invasiveness of DU145 in Matrigel. (B) Numbers of DU145/XB130- cells that pass through Matrigel was significantly less than control group. (C) XB130 downregulation suppressed invasiveness of LNCap in Matrigel. (D) Numbers of LNCap/XB130- cells that pass through Matrigel was significantly less than control group. Data are represented as mean $+/-\mathrm{SEM} . * P<0.05$ as compared to control groups. $* * P<0.01$ as compared to control groups. 
that XB130 expression may promote metastasis of prostate cancer.

Abnormal activation of PI3K/AKT pathway has been implicated in anti-apoptosis and dysplasia of many malignancies like colon caner, non-small cell lung carcinoma and lymphoma [19]. A YxxM motif in the $\mathrm{N}$-terminal of XB130 facilitates the interaction with $\mathrm{p} 85$ subunit of PI3K, and therefore stimulates AKT activity in both thyroid and lung cancer cells [20]. Furthermore, downregulation of XB130 in thyroid cancer cells leads to decreased Akt phosphorylation at residue 473, which in turn inhibits cell cycle progression and cell growth [9]. In present analysis of PI3K/AKT pathway in DU145/XB130and LNCap/XB130- cells, XB130 knockdown was accompanied by reduced expression of p-PDK1, thr308, $\mathrm{p}-\mathrm{C}$-Raf and upregulation of $\mathrm{p}$-PTEN, which indicates that reducing XB130 deters Akt pathway in prostate cancer as in other cancers. Given the fact that AKT plays a key

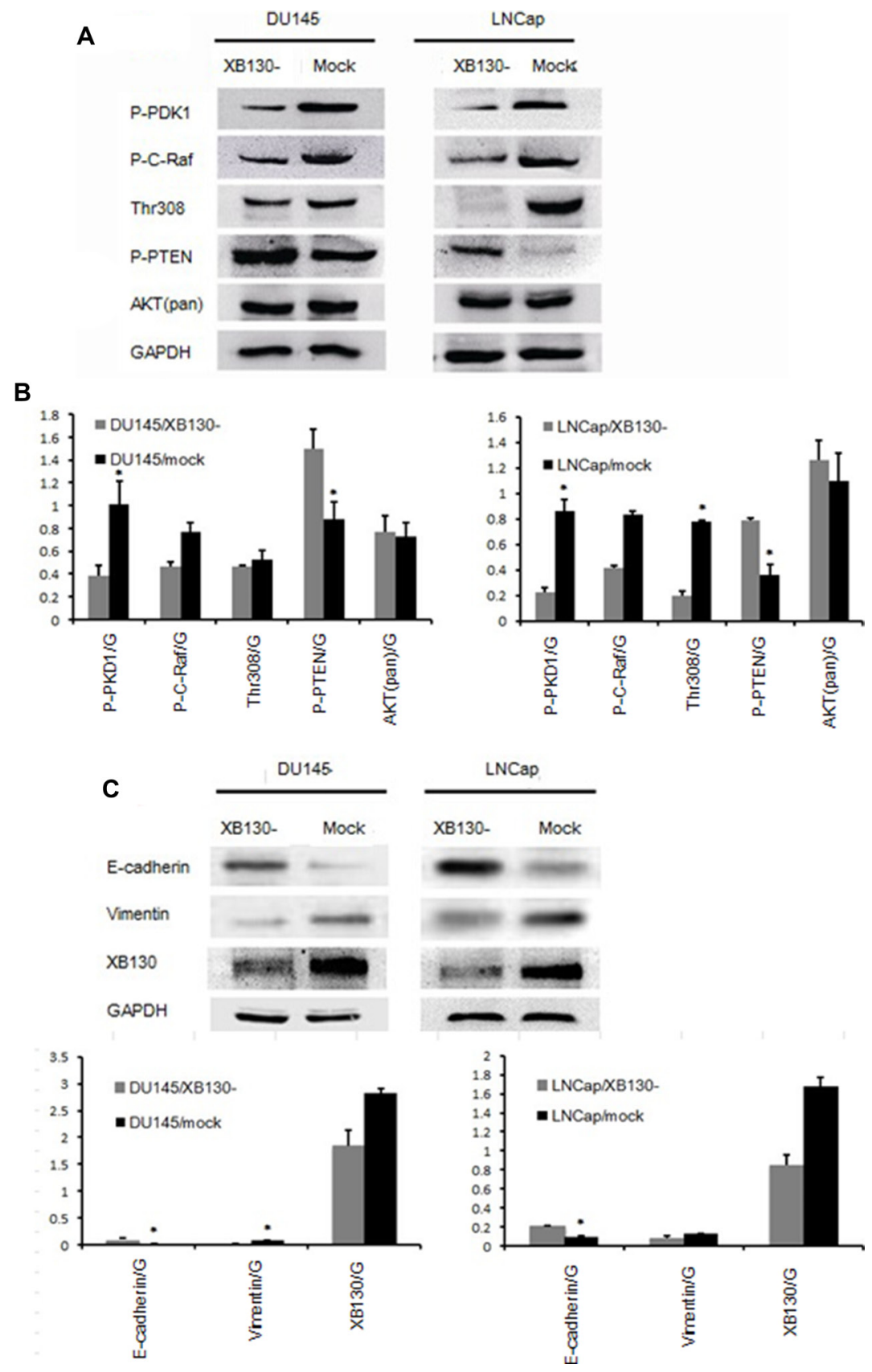

Figure 6: XB130 was involved in both Akt signaling and EMT process. (A) Akt signaling altered with XB130 interference in both DU145 and LNCap. (B) Semi quantitative analysis of protein level changes in Akt pathway in DU145/XB130- and LNCap/XB130-. (C) EMT process was hindered by XB130 knockdown. (D) Semi quantitative analysis of protein level changes in EMT process in DU145/ $\mathrm{XB130}$ - and LNCap/XB130-. Data are represented as mean $+/-$ SEM. $* P<0.05$ as compared to control groups. 
Table 2: Oligonucleotide sequences of shRNAs targeting XB130

\begin{tabular}{|l|l|l|}
\hline shXB130-A & Sense & $\begin{array}{l}\text { GATCCCCGGAGCTAAAGGAAACCCTACTTTCAAGAGAAGTAGGGTTTCCTTTAG } \\
\text { CTCCTTTT }\end{array}$ \\
\cline { 2 - 3 } & Antisense & $\begin{array}{l}\text { AGCTTAAAAAGGAGCTAAAGGAAACCCTACTTCTCTTGAAAGTAGGGTTTCCTT } \\
\text { TAGCTCCGGG }\end{array}$ \\
\hline shXB130-B & Sense & $\begin{array}{l}\text { GATCCCCGCCGATAGGGTCTCCTGTATTTTCAAGAGAAATACAGGAGACCCTATC } \\
\text { GGCTTTTT }\end{array}$ \\
\hline shXB130-C & Sense & $\begin{array}{l}\text { GATCCCCGCTGAAGATCACACCGATGTTCAAGAGACATCGGTGTGATCTTCAGC } \\
\text { GCTCCGGG }\end{array}$ \\
\cline { 2 - 3 } & Antisense & $\begin{array}{l}\text { AGCTTAAAAAGCTGAAGATCACACCGATGTCTCTTGAAAGTAGGGTTTCCTTTAG } \\
\text { CTCCGGG }\end{array}$ \\
\hline $\begin{array}{l}\text { XB130- } \\
\text { scramble }\end{array}$ & Sense & $\begin{array}{l}\text { GATCCCCGCCAGCTTAGCACTGACTCTTCAAGAGAGAGTCAGTGCTAAGCTGG } \\
\text { CTTTTT }\end{array}$ \\
\cline { 2 - 3 } & Antisense & $\begin{array}{l}\text { AGCTTAAAAAGCCAGCTTAGCACTGACTCTCTCTTGAAAGTAGGGTTTCCTTTA } \\
\text { GCTCCGGG }\end{array}$ \\
\hline
\end{tabular}

role in the cellular proliferation and survival and XB130 knockdown impedes AKT pathway, we concludes that XB130 downregulation may prohibit cell growth through inhibiting PI3K/AKT in prostate cancer.

PI3K/Akt signaling has been proved to be closely related with EMT events [21]. XB130 overexpression in squamous cancer cells SCC13 and SCC15 urge epithelial cells to gain characteristics of fibroblast, with decreased E-Cadherin and $\beta$-catenin and increased vimentin, which indicated occurrence of EMT event, reduction of cellular attachment and augmentation of mobility and invasiveness [22]. E-Cadherin was also reduced in DU145/XB130- and LNCap/XB130- associated with enhancement of vimentin expression, indicated that XB130 may promote migration and invasion of prostate cancer through mediating EMT by activating PI3K/Akt.

\section{MATERIALS AND METHODS}

\section{Human specimens and cell lines}

Two hundreds and ten paraffin-embedded prostate cancer samples and clinical information from April, 2000 to September, 2013 were obtained from consenting patients in General Hospital of Guangzhou Military Command of People's Liberation Army. The age range was 15 to 83 years. The experimental procedures were approved by the Research Ethics Committee of General Hospital of Guangzhou Military Command of People's Liberation Army.

Human prostate cancer cell line 22RV1, DU145, LNCap and human embryonic kidney cell line 293FT cells were purchased from Shanghai Cell Bank of Chinese Academy of sciences. 22RV1 and LNCap were cultured in RPMI-1640 with $10 \%$ fetal calf serum. DU145 and PC3 were cultured in F-12 medium with 10\% fetal bovine serum. All cell lines were incubated in a $37^{\circ} \mathrm{C}$ incubator with $5 \% \mathrm{CO} 2$.

\section{Immunohistochemistry (IHC)}

IHC was performed with rabbit anti-XB130 polyclonal antibody (Abnova) and EnVisionTM plus kit (DAKO) according to manufacturer's instruction. Briefly, after deparaffinization and hydration, retrieve antigen with citrate buffer ( $\mathrm{pH}$ 6.0) and quenched endogenous peroxidase activity with $0.3 \%$ hydrogen peroxide solution, the sections were incubated with primary antibody (anti-XB130, 1:100) for 2 hours at room temperature. After incubation with secondary antibody for 30 minutes at room temperature, the bound antibody was detected with DAB as substrate. As positive control, sections with vascular epithelium were immunostained. Sections stained with PBS instead of primary antibody were applied as negative control. We used an IHC scoring system from Engers et al. Two independent pathologists, both masked to the patients' clinical status, made these judgments.

\section{Knockdown of XB130 in DU145 and LNcap}

DNA constructs for expression of V5- and GFP epitope tagged CycG2 fusion proteins in mammalian cells have been described $(13,15)$. Selection of shRNA target sites was done with RNAiDesignsoftware (Invitrogen). Oligonucleotide sequences are as described in Table 2.

ShRNAs were inserted into linearized pGCSILGFP at AgeI and EcoRI sites. Lentiviruses were produced by transfecting 293FT cells of pGCSIL-GFP constructs containing shRNAs, pHelper 1.0 and pHelper 2.0 with Lipo2000 (Invitrogen). Supernatants were filtered and used for infecting DU145 and LNcap. Infection efficacy 
was observed by GFP expression under fluorescence microscope. Cell lines with over $80 \%$ efficacy were considered stable.

\section{RT-PCR}

Total RNA was extracted with Trizol reagent (Invitrogen) and reversely transcribed according to the manufacturer's instruction (Invitrogen). Semi quantitative reverse transcription-polymerase chain reaction was used to measure XB130 mRNA levels using primers XB130-F (CTCCTCCGGCTTTACACCAAA) and XB130-R (GGCAAGCTGTTTCCGTTCTG), with GAPDH as control (GAPDH-F, TGTGGGCATCAATGGATTTGG; GAPDH-R, TGTGGGCATCAATGGATTTGG).

\section{Western blot}

Cells were lysed in RIPA buffer and quantified with a BCA Protein Assay Reagent Kit (Pierce). 20 ug Cell lysates of total proteins were separated by SDS-PAGE, and transferred onto PVDF membranes (Roche). Blot then was probed with indicated antibodies and detected with an enhanced chemiluminescence detection kit (ECL) (Thermo). Band density was quantified using the ImageJ software (http://rsb.info.nih.gov/ij/). Rabbit polyclonal antibodies against XB130 (1:1000, PGT), pan-AKT (1:1000, Cell signaling), p-PDK1 (1:1000, Cell signaling), p-c-Raf (1:1000, Cell signaling), Thr308 (1:1000, Cell signaling), p-PTEN (1:1000, Cell signaling), p-GSK (1:1000, Cell signaling), E-cadherin (1:1000, Cell signaling), Vimentin (1:1000, Cell signaling) and mouse monoclonal antibodies against tubulin (1:4000, PGT) were used.

\section{Viability assay}

For CCK8 assays, $5 \times 10^{3}$ cells were plated in 96well plates and observed for viability for 7 days. CCK8 method was used to detect the viability of cells according to the manufacturer's instructions. The absorbance at $570 \mathrm{~nm}$ was measured using a microplate reader. Five replicate wells were used for each group.

\section{Colony formation assay}

For colony formation assay, 100 single cells from each group were seeded in 6-well plates and cultured at $37^{\circ} \mathrm{C}$ with $5 \% \mathrm{CO} 2$ for 14 days before visualized by Giemsa stain. Colonies containing $>50$ cells were scored, efficiency was calculated by the percentage of colony numbers in seeded cell numbers.

\section{Cell cycle analysis}

Cells were fixed in $75 \%$ ethanol at $-20^{\circ} \mathrm{C}$ for overnight. Washed pellets of fixed cells were resuspended in PBS containing 0.1\% RNaseA (Fermentas) and $100 \mathrm{ul}$ $10 \mathrm{ug} / \mathrm{ml}$ propidium iodide (Sigma) for $30 \mathrm{~min}$ at room temperature before flow cytometry using a FACScan (BD Biosciences) as described.

\section{Transwell assay}

Cells were plated on the top matrigel-coated chamber of 24-well plate in medium without serum. Medium supplemented with 600 ul 10\% FBS was used in the bottom chamber. After incubation at $37^{\circ} \mathrm{C}$ with $5 \%$ $\mathrm{CO} 2$ for 48 hours, cells on the lower membrane were fixed in $100 \%$ methanol and stained. Five random visual fields of each insert were counted under a microscope. Three independent experiments were conducted and the data were presented as the means \pm SEM.

\section{In vivo xenograft animal model}

$\mathrm{BALB} / \mathrm{c}$ athymic nude mice were purchased from veterinary facilities of Southern Medical University. The mice were housed and maintained in accordance with the Animal Care and Use Guidelines of Southern Medical University under a protocol approved by the animal ethics committee of Southern Medical University. $10^{7}$ cells were trypsinized and washed into single cell suspension with serum-free medium. DU145/XB130- paired with DU145/ mock and LNCap/XB130- paired with LNCap/mock were inoculated in nude mice respectively (XB130 knockdown cells on the right flank and control on the left counterpart). Mice were observed for 24 days before sacrifice by cervical dislocation. Tumors were measured every three days, then excised and fixed in formaldehyde. Six mice were in each group.

\section{Statistical methods}

Correlation between XB130 expression and survival was analyzed with Kaplan-Meier method. RT-PCR, CCK8, cell cycle and transwell assay were compared with one-way ANOVA. T-test was used to compare Western blot, colony formation assay and tumor volumes. $\chi^{2}$ tests were conducted to assess correlations between XB130 expression and increase prostate specific antigen (PSA), free PSA (f-PSA), prostatic acid phosphatase (PAP) and $\mathrm{T}$ classification.

\section{CONFLICTS OF INTEREST}

We declare that we have no financial and personal relationships with other people or organizations that can 
inappropriately influence our work, there is no professional or other personal interest of any nature or kind in any product, service and/or company that could be construed as influencing the position presented in, or the review of, the manuscript entitled, "XB130 is overexpressed in prostate cancer and involved in cell growth and invasion".

\section{GRANT SUPPORT}

1. Science and Technology Planning Project of Guangdong Province (No.2014A020212260); 2. Major Projects for Collaborative Innovation in Medical Health Care (Contract No.201508020253).

\section{REFERENCES}

1. Jemal A, Bray F, Center MM, Ferlay J, Ward E, Forman D. Global cancer statistics. CA Cancer J Clin. 2011; 61:69-90.

2. Siegel R, Naishadham D, Jemal A. Cancer statistics, 2012. CA Cancer J Clin. 2012; 62:10-29.

3. Gilbert SM, Cavallo CB, Kahane H, Lowe FC. Evidence suggesting PSA cutpoint of $2.5 \mathrm{ng} / \mathrm{mL}$ for prompting prostate biopsy: review of 36,316 biopsies. Urology. 2005; 65:549-553.

4. Bouton AH, Riggins RB, Bruce-Staskal PJ. Functions of the adapter protein Cas: signal convergence and the determination of cellular responses. Oncogene. 2001; 20:6448-6458.

5. Cherezova L, Gatesman A, Flynn DC. The effects of phosphorylation on adaptor protein function. Front Biosci. 2002; 7:d164-203.

6. Alexandropoulos K, Baltimore D. Coordinate activation of c-Src by SH3- and SH2-binding sites on a novel p130Casrelated protein, Sin. Genes Dev. 1996; 10:1341-1355.

7. Xu J, Bai XH, Lodyga M, Han B, Xiao H, Keshavjee S, Hu J, Zhang H, Yang BB, Liu M. XB130, a novel adaptor protein for signal transduction. J Biol Chem. 2007; 282:16401-16412.

8. Grupe A, Li Y, Rowland C, Nowotny P, Hinrichs AL, Smemo S, Kauwe JS, Maxwell TJ, Cherny S, Doil L. A scan of chromosome 10 identifies a novel locus showing strong association with late-onset Alzheimer disease. Am J Hum Genet. 2006; 78:78-88.

9. Lodyga M, De Falco V, Bai X, Kapus A, Melillo R, Santoro M, Liu M. XB130, a tissue-specific adaptor protein that couples the RET/PTC oncogenic kinase to PI 3-kinase pathway. Oncogene. 2009; 28:937-949.

10. Shiozaki A, Lodyga M, Bai XH, Nadesalingam J, Oyaizu T, Winer D, Asa SL, Keshavjee S, Liu M. XB130, a novel adaptor protein, promotes thyroid tumor growth. Am J Pathol. 2011; 178:391-401.
11. Monika L, Xiao-Hui B, András K, Mingyao L. Adaptor protein $\mathrm{XB130}$ is a Rac-controlled component of lamellipodia that regulates cell motility and invasion. J Cell Sci. 2010; 123:4156-4169.

12. Grieco M, Santoro M, Berlingieri MT, Melillo RM, Donghi R, Bongarzone I, Pierotti MA, Della Ports G, Fusco A, Vecchiot G. PTC is a novel rearranged form of the ret protooncogene and is frequently detected in vivo in human thyroid papillary carcinomas. Cell. 1990; 60:557-563.

13. Shi M, Huang W, Lin L, Zheng D, Zuo Q, Wang L, Wang N, Wu Y, Liao Y, Liao W. Silencing of XB130 is associated with both the prognosis and chemosensitivity of gastric cancer. PLoS One. 2012; 7:e41660.

14. Zhang J, Jiang X. Prognostic significance of XB130 expression in surgically resected pancreatic ductal adenocarcinoma. World J Surg Oncol. 2014; 12:49.

15. Shiozaki A, Kosuga T, Ichikawa D, Komatsu S, Fujiwara H, Okamoto K, Iitaka D, Nakashima S, Shimizu H, Ishimoto T. $\mathrm{XB130}$ as an independent prognostic factor in human esophageal squamous cell carcinoma. Ann Surg Oncol. $2013 ; 20: 3140-3150$.

16. Zhang J, Jiang $X$, Zhang J. Prognostic significance of XB130 expression in surgically resected pancreatic ductal adenocarcinoma. World J Surg Oncol. 2014; 12:1-5.

17. Li J, Sun W, Wei H, Wang X, Li H, Yi Z. Expression of XB130 in human ductal breast cancer. Int J Clin Exp Patho. 2015; 8:5300.

18. Wu Q, Nadesalingam J, Moodley S, Bai X, Liu M. XB130 translocation to microfilamentous structures mediates NNK-induced migration of human bronchial epithelial cells. Oncotarget. 2015; 6:18050-65. doi: 10.18632/ oncotarget. 3777.

19. Katso R, Okkenhaug K, Ahmadi K, White S, Timms J, Waterfield MD. Cellular function of phosphoinositide 3-kinases: implications for development, immunity, homeostasis, and cancer. Annu Rev Cell Dev. 2001; 17:615-675.

20. Shiozaki A, Shen-Tu G, Bai X, Iitaka D, De Falco V, Santoro M, Keshavjee S, Liu M. XB130 mediates cancer cell proliferation and survival through multiple signaling events downstream of Akt. PLoS One. 2012; 7:e43646.

21. Larue L, Bellacosa A. Epithelial-mesenchymal transition in development and cancer: role of phosphatidylinositol 3 'kinase/AKT pathways. Oncogene. 2005; 24:7443-7454.

22. Grille SJ, Bellacosa A, Upson J, Klein-Szanto AJ, Van Roy F, Lee-Kwon W, Donowitz M, Tsichlis PN, Larue L. The protein kinase Akt induces epithelial mesenchymal transition and promotes enhanced motility and invasiveness of squamous cell carcinoma lines. Cancer Res. 2003; 63:2172-2178. 